\title{
Estresse de alunos de escola pública no pré-vestibular e a influência do estilo parental
}

Stress of public school students in the pre-university entrance exam and the influence of pariental style

\section{Estrés de los estudiantes de escuelas públicas en el examen de acceso preuniversitario y la influencia del estilo pariental}

Hatiro Antonio Marcelo Narazaki ${ }^{1 *}$, Felipe Tebaldi de Souza Melotti ${ }^{1}$, Jefferson Kenkiti kkuno $^{1}$, Júlia Maria da Matta ${ }^{1}$, Nadjila Otsuka Amad Kfouri ${ }^{1}$, Rafael Suleiman Zarate de Lima ${ }^{1}$, Vagnum Dias da Silva ${ }^{2}$.

\section{RESUMO}

Objetivo: Avaliar o nível de estresse de alunos de escolas públicas estudantes em cursinhos pré-vestibulares populares, correlacionando a influência do estilo parental ao evento estressor vestibular. Métodos: Trata-se de um estudo descritivo de levantamento normativo que envolveu 113 alunos de escola pública estudantes de cursinhos populares. Os instrumentos aplicados foram as escalas de stress para adolescentes e de responsividade e exigência parental. Resultados: A amostra é de 96 mulheres (85\%) e 13 homens (15\%). Os resultados mostram a prevalência de estresse no sexo feminino com escore de 3,10, próximo ao valor considerado estressado, já a amostra masculina obteve escore 2,19, não apresentando nível de estresse relevante no estudo. Além disso, os sintomas cognitivos e fisiológicos de estresse têm alta prevalência nas mulheres, com 72,9\% e 63,5\% respectivamente, indicando maiores problemas de saúde nesse grupo. Por fim, os dois grupos em geral são altamente responsivos e com níveis baixos de exigência. Conclusão: Conclui-se que o estresse é muito prevalente em vestibulandos de cursinhos populares oriundos de escolas públicas, tornando-o um problema de saúde a ser monitorado, e que, no âmbito familiar, pode haver melhora nos sintomas do estresse caso haja uma relação de caráter mais responsivo.

Palavras-chave: Estresse, Vestibular, Saúde, Adolescente.

\begin{abstract}
Objective: Evaluate the stress level of public school students attending popular pre-university courses, correlating the influence of parenting style to the stressful event. Methods: This is a cross-sectional and correlational study involving 113 public school students who are students of popular courses. The instruments applied were the stress scales for adolescents and the parental responsiveness and demand scales. Results: Sample is 96 women $(85 \%)$ and 13 men (15\%). The results show the prevalence of stress in females with a score of 3.10, close to the value considered stressed, whereas the male sample obtained a score of 2.19 , with no relevant level of stress in the study. In addition, cognitive and physiological stress symptoms are highly prevalent in women, with $72.9 \%$ and $63.5 \%$ respectively, indicating greater health problems in this group. Finally, both groups are generally highly responsive and have low levels of demand. Conclusion: It is concluded that stress is very prevalent in college entrance exams for popular courses from public schools, making it a health problem to be monitored, and that, in the family context, there may be an improvement in stress symptoms if there is a relationship of character more responsive.
\end{abstract}

Keywords: Stress, Entrance exam, Health, Adolescent.

1 Universidade Mogi das Cruzes (UMC), Mogi das Cruzes - SP. *E-mail: hatiromarcelo@gmail.com

2 Universidade Federal de São Carlos (UFSCAR), São Carlos - SP. 


\section{RESUMEN}

Objetivo: Evaluar el nivel de estrés de los estudiantes de escuelas públicas que asisten a cursos universitarios preuniversitarios populares, correlacionando la influencia del estilo de crianza con el evento estresante. Métodos: Este es un estudio transversal y correlacional que involucra a 113 estudiantes de escuelas públicas que son estudiantes de cursos populares. Los instrumentos aplicados fueron las escalas de estrés para adolescentes y las escalas de respuesta y demanda de los padres. Resultados: La muestra es de 96 mujeres $(85 \%)$ y 13 hombres (15\%). Los resultados muestran la prevalencia de estrés en mujeres con una puntuación de 3,10 , cercana al valor considerado estresado, mientras que la muestra masculina obtuvo una puntuación de 2,19, sin un nivel de estrés relevante en el estudio. Además, los síntomas de estrés cognitivo y fisiológico son muy prevalentes en las mujeres, con un $72,9 \%$ y un $63,5 \%$ respectivamente, lo que indica mayores problemas de salud en este grupo. Por último, ambos grupos son en general muy receptivos y tienen bajos niveles de demanda. Conclusión: Se concluye que el estrés es muy prevalente en los exámenes de ingreso a cursos populares de las escuelas públicas, lo que lo convierte en un problema de salud a monitorear, y que, en el contexto familiar, puede haber una mejora en los síntomas del estrés si existe una relación de personaje más receptivo.

Palabras clave: Estrés, Examen de ingreso, Salud, Adolescente.

\section{INTRODUÇÃO}

O ser humano é uma realidade biopsicossocial. Tal afirmação surge da premissa de que o entendimento integral da condição humana só pode ser alcançado quando levados em conta três abordagens: a biológica, a psicológica e a social. Trata-se de uma mudança na relação saúde-doença, configurando o paradigma biopsicossocial (PEREIRA TTSO, et al., 2011). Nesse sentido, a adolescência, definida no Brasil pelo marco legal do Estatuto da Criança e do Adolescente (ECA) (2017) como o período compreendido entre 12 e 18 anos, precisa ser vista como um emaranhado de fatores de ordem individual, por estar associada à maturidade biopsicossocial, que acontece rapidamente (JUSTO AP, 2005; SILVA LLT, et al., 2020).

Cada sujeito, em suas dimensões biológica, psicológica e social, constitui uma unidade inseparável. Sendo assim, a atenção à saúde desse grupo deve pautar-se na integralidade, levando em consideração sua adaptação ao ambiente, suas relações com diversas organizações sociais e seus hábitos e comportamentos que, em determinadas conjunturas, podem vulnerabilizá-lo e impactar sua saúde (MINISTÉRIO DA SAÚDE, 2017). A adolescência é um período de grandes mudanças, no qual há diversas cobranças sociais, mudanças corporais e emocionais, além de incertezas em relação ao futuro (PAGGIARO P, et al., 2009).

Como uma fase de transição, ela é marcada, portanto, por eventos estressores (MACHADO SF, et al., 2011). Somado a isso, há outros fatores que podem contribuir para desencadear e/ou manter o stress, como estrutura familiar, estilo parental, saúde do adolescente, condição socioeconômica da família, etc. (JUSTO AP, 2005; SILVA LLT, et al., 2020). Desse modo, o ambiente de mudanças no qual o adolescente está inserido demanda grau elevado de ajustes para que ele se adeque aos modelos estabelecidos, gerando um desequilíbrio entre sua capacidade pessoal e as demandas do ambiente (MARQUES CP, et al., 2015).

Para entender a concepção de estresse é necessário, sobretudo, recorrer ao conceito de homeostase. A homeostase corresponde à capacidade de autorregulação que o organismo tem para manter a estabilidade do seu meio interno (OLIVEIRA EA, 2006). O termo estresse, a partir disso, é definido como uma resposta psicofisiológica complexa que provoca excitação emocional e perturba a homeostasia. (LIPP MEN, 2003; AMARAL SCO e SANT'ANNA CC, 2018).

No fenômeno do estresse, é necessário considerar ainda outras variáveis, como a avaliação cognitiva (avalição inicial da situação, avaliação da demanda da situação, avaliação de capacidade e seleção da resposta) e as respostas diante de um estressor (enfrentamento/ataque, evitação/fuga, passividade /colapso) (SELYE H, 1965; STRAATMANN G, 2010).

$\mathrm{Na}$ avaliação do nível cognitivo, o sujeito inicialmente avalia o potencial de ameaça do estímulo/da situação, depois avalia a demanda da situação de acordo com seu histórico pessoal e suas experiências 
prévias, medindo, em seguida, a sua capacidade para lidar e enfrentar a situação, por fim, a partir do levantamento anterior o sujeito elabora suas respostas às demandas apreendidas. No que diz respeito, as respostas diante de um estressor, a seleção do tipo de enfretamento a ser feito pelo indivíduo é o que definirá a ativação de recursos e estruturas fisiológicas específicas e os possíveis transtornos psicofisiológicos advindos dessa escolha (SELYE H, 1965; STRAATMANN G, 2010).

Nesse sentido, considerando o adolescente como um ser integrado às diversas entidades sociais, as interações socializadoras na família exercem papel fundamental na sua vulnerabilidade ao estresse, já que o apoio dos pais e de pares é fundamental para que o adolescente lide melhor com fatores estressores (JUSTO AP, 2005). O estilo parental, representado pela exigência e responsividade dos pais, acompanhado pelo medo do fracasso e de más decisões, exerce grande pressão sobre o estudante, que muitas vezes vê o processo seletivo do vestibular como algo angustiante pelo seu caráter avaliativo, no qual a capacidade intelectual de anos é medida em uma única prova (PAGGIARO P, et al., 2009; SCHÖNHOFEN FT, et al., 2020).

No tocante aos pais, as características da exigência parental referem-se às atitudes que buscam controlar o comportamento dos filhos como limites e regras. Nesse controle (exigência) dos pais, estão inclusas as condutas frequentes de cobrança, vigilância, monitoramento e disciplina. A responsividade, por outro lado, refere-se à afetividade e à comunicação dos pais para com os filhos, geradas a partir de atitudes compreensivas e que atendem às demandas e singularidades dos filhos, tendo como fim criar autonomia $e$ autoconfiança aos jovens (DARLING N e STEINBERG L, 1993; TEIXEIRA MAP, 2004; VARGAS P, et al., 2017).

Já no que se refere ao processo seletivo, o exame vestibular, meio de ingresso para o ensino superior para milhares de alunos no Brasil, caracteriza-se por ser um período de tensão estressora prolongada, já que a sua preparação dura, em média, um ano, com alto índice de concorrência (SANTOS FS, et al., 2017). No Brasil, anualmente, o Exame Nacional do Ensino Médio (ENEM), é a principal forma de exame vestibular, pois oferece bolsas integrais ou parciais em universidades particulares, Programa Universidade para Todos (PROUNI) ou vagas no ensino superior público, Sistema de Seleção Unificada (SiSU), aos estudantes (MARQUES BAA, 2013).

Dentro dessa abordagem, os cursinhos pré-vestibulares populares (comunitários) são projetos que recebem muitos adolescentes do ensino público, que procuram preparação e incentivo para ingressarem no Ensino Superior. Esses cursinhos, por estarem localizados principalmente nas periferias da Grande São Paulo, e como consequência das políticas de ações afirmativas (SiSU e PROUNI), têm conseguido o acesso à Educação Superior para camadas sociais pouco representadas historicamente nesse nível de ensino (RIMOLI J, et al., 2020; ARAGÃO RC, et al., 2015).

Para buscar medidas de enfrentamento ao estresse dos vestibulandos nesses cursinhos, além de auxiliálos para um melhor desempenho nas provas, seria importante elucidar os efeitos do estilo parental na prevenção de males relacionados ao estresse, como depressão e suicídio, já relatados na literatura (LANDSTEDT E e GADIN KG, 2012; SCHERMANN LB, et al., 2014; GROLLI V, et al., 2017).

O presente trabalho teve por objetivo investigar o nível de estresse dos vestibulandos adolescentes de 02 cursinhos populares oriundos de escolas públicas, relacionando-o com o estilo parental, um fator determinante para o enfrentamento do estresse do adolescente vestibulando. Como objetivo secundário, a pesquisa busca confirmar os resultados obtidos em outras pesquisas em relação à variável sexo, nas quais os resultados obtidos mostram um maior índice de estresse relacionado às mulheres.

\section{MÉTODOS}

Trata-se de um estudo descritivo de levantamento normativo que envolveu 113 adolescentes alunos de escola pública estudantes de dois cursinhos populares na periferia da grande São Paulo.

Os critérios de inclusão para pesquisa foram: estar dentro da faixa etária de 12 a 18 anos, sugerida pela ECA para definição de adolescência; estar matriculado em algum dos dois cursinhos populares; ser aluno oriundo de escola pública; morar (conviver) com o pai e a mãe, sejam eles biológicos ou substitutos; aceitar 
participar da pesquisa, com o consentimento dos pais, por meio da assinatura do Termo De Consentimento Livre e Esclarecido (TCLE), ou pela assinatura do Termo de Assentimento Livre e Esclarecido (TALE) para os adolescentes com 18 anos de idade.

Foram excluídos da pesquisa menores de 12 anos e maiores de 18 anos, alunos que não convivam com os pais, alunos oriundos de escolas particulares e adolescentes que não enviaram o termo (TCLA e TALE) com as devidas assinaturas.

Com o auxílio de uma psicóloga, foram aplicados os questionários de Escala de Stress para Adolescentes, uma versão do Inventário da Ansiedade Traço-Estado (DATE), e de Escala de Responsividade e Exigência Parental, TRICOLI VAC (2006), via Google Forms, aos alunos, de modo a determinar e correlacionar os fatores de apoio intrafamiliar e os níveis de estresse e ansiedade apresentados pelos estudantes.

O presente projeto, previamente autorizado pelos cursinhos, teve a aprovação em Comitê de Ética em Pesquisa, com o número de CAAE 25080919.1.0000.5504, e não envolveu riscos físicos, uma vez que não houve processos invasivos. Como havia a possibilidade de desconfortos psicológicos, emocionais e culturais, foi comunicado previamente aos alunos sobre a possibilidade de não precisarem responder àquilo que não se sentiam à vontade ou desistir da participação.

\section{RESULTADOS}

Após a realização dos questionários, o espaço amostral obtido foi de 113 estudantes, com média de idade de 17,6 anos ( $(\mathrm{PP}=0,7)$, nos quais $15 \%$ são do sexo masculino e $85 \%$ do sexo feminino, evidenciando um predomínio do sexo feminino na amostra estudada, sendo que $92,9 \%$ do total da amostra coabita com os pais (Tabela 1).

Tabela 1 - Caracterização dos vestibulandos de cursinhos populares oriundos de escolas públicas, $n=113$.

\begin{tabular}{ccc}
\hline Variável & N & $\%$ \\
\hline Sexo & & \\
\hline Masculino & 17 & 15 \\
Feminino & 96 & 85 \\
\hline Idade & & \\
\hline 18 anos & 83 & 73,5 \\
17 anos & 17 & 15 \\
16 anos & 13 & 11,5 \\
\hline Mora com a mãe e com o pai & & \\
\hline Não & 8 & 7,1 \\
Sim & 105 & 92,9 \\
\hline Total & $\mathbf{1 1 3}$ & $\mathbf{1 0 0}$ \\
\hline
\end{tabular}

Fonte: Narazaki HAM, et al., 2021.

Por fim, a classificação de estresse foi obtida por meio do cálculo do escore para a Escala de Stress para Adolescentes (ESA), que indicou grupo estressado maior que 3,11 para meninas e maior que 2,64 para meninos. O grupo masculino obteve escore de 2,19; dessa forma, os meninos foram classificados como grupo não estressado, já o grupo feminino apresentou escore de 3,10, e apesar de menor que a média considerada, como há uma mínima diferença e existem sintomas de estresse, o grupo foi considerado estressado.

Como o grupo das mulheres foi classificado dessa forma, foi calculado o escore dos quatros domínios (psicológico, cognitivo, fisiológico e interpessoal), como orientado no questionário, o qual foi considerado maior que 3,50 para sintoma psicológico, maior que 2,83 para sintoma cognitivo, maior que 2,89 para sintoma fisiológico e, por fim, maior que 2,60 para sintoma interpessoal. Caso a média calculada fosse superior aos cortes propostos, classificar-se-ia o estresse de acordo com seu respectivo domínio.

Mediante tais valores de referência, obteve-se que o grupo feminino apresentou padrão de estresse com sintomatologia fisiológica e cognitiva, devido ao escore calculado corresponder a 3,07, no caso dos sintomas 
fisiológicos, e 3,29 para a sintomatologia cognitiva. Ainda sobre a classificação de estresse, 72,9\% das meninas apresentaram escore cognitivo maior que 2,83 , enquanto $63,5 \%$ apresentaram escore fisiológico superior a 2,89.

Ademais, em relação à análise do controle (exigência) e da responsividade (cuidado) parental, em ambos os grupos (masculino e feminino) observou-se que $54 \%$ dos pais têm alta exigência e $46 \%$ baixa exigência. Com relação à responsividade, 52,2\% apresentaram alta responsividade e 47,8\% baixa (Tabela 2).

Ainda baseado na Tabela 2, classificou-se como alta responsividade ou exigência a média maior que 2 , e, caso ela fosse menor que 2 , considerou-se baixa responsividade ou exigência, devido a mediana equivaler a 2. Portanto, tem-se que, de modo geral, o grupo possui ambos os pais com alta responsividade e baixa exigência.

Tabela 2 - Controle e da responsividade parental, nos grupos masculino e feminino, $n=113$.

\begin{tabular}{cccccc}
\hline Variável & Alta & Baixa & Média & Desvio Padrão & Mediana \\
\hline Exigência & $54 \%$ & $45 \%$ & 1,99 & 1,56 & 2 \\
\hline Responsividade & $52,2 \%$ & $47,8 \%$ & 2,05 & 1,57 & 2 \\
\hline
\end{tabular}

Fonte: Narazaki HAM, et al., 2021.

Baseado na classificação do grupo em geral, foi possível aprofundar e ramificar a análise, por meio da divisão em sexo masculino e feminino, pais e mães, classificando a responsabilidade e exigência em alta ou baixa. Dessa forma, caso fosse observado o nível de exigência entre os sexos, dividindo-os entre pais e mães, tem-se que, no sexo feminino, $58,3 \%$ das meninas afirmam alto nível de exigência materna, enquanto $41,7 \%$ das meninas afirmam baixo nível de exigência materna (Tabela 3).

Em relação à exigência paterna do sexo feminino, tem-se na Tabela 3 que 45,8\% das meninas consideram alto nível de exigência por parte dos pais, enquanto $54,2 \%$ das meninas consideram baixa exigência. Em linhas gerais, maior parcela das mães é considerada altamente exigente, enquanto menor parcela dos pais é considerada altamente exigente.

Perante o sexo masculino, obteve-se que $82,3 \%$ dos meninos consideram alta exigência materna, enquanto $17,7 \%$ dos meninos consideram baixa exigência. Já em relação à exigência paterna, observa-se que $47 \%$ dos meninos consideram alta exigência paterna, enquanto $53 \%$ consideram baixa exigência. $\mathrm{Da}$ mesma forma que no sexo feminino, os homens consideram a maior parte das mães como altamente exigente, enquanto os pais com alta exigência representam menor parcela (Tabela 3).

Tabela 3 - Exigência parental.

\begin{tabular}{|c|c|c|c|c|c|c|c|c|}
\hline \multirow{3}{*}{ Variável } & \multicolumn{4}{|c|}{ Alunas } & \multicolumn{4}{|c|}{ Alunos } \\
\hline & \multicolumn{2}{|c|}{ Mãe } & \multicolumn{2}{|c|}{ Pai } & \multicolumn{2}{|c|}{ Mãe } & \multicolumn{2}{|c|}{ Pai } \\
\hline & $\mathbf{N}$ & $\%$ & $\mathbf{N}$ & $\%$ & $\mathbf{N}$ & $\%$ & $\mathbf{N}$ & $\%$ \\
\hline \multicolumn{9}{|l|}{ Média } \\
\hline$>2^{*}$ & 56 & 58,3 & 44 & 45,8 & 14 & 82,3 & 8 & 47 \\
\hline$<2^{* *}$ & 40 & 41,7 & 52 & 54,2 & 3 & 17,7 & 9 & 53 \\
\hline Total & 96 & 100 & 96 & 100 & 17 & 100 & 17 & 100 \\
\hline
\end{tabular}

Por fim, com relação à responsividade parental, analisando a Tabela 4, a seguir, obteve-se que, para o sexo feminino, $66,6 \%$ das alunas consideraram as mães com alta responsividade, enquanto $33,4 \%$ consideraram baixo nível dessa variável. Com relação aos pais, tem-se que $37,5 \%$ das alunas consideram os pais com alta responsividade, enquanto $62,5 \%$ consideram-nos com baixa. Portanto, a maioria das mães é considerada altamente responsiva, enquanto os pais altamente responsivos são minoria. 
Tabela 4 - Responsividade parental.

\begin{tabular}{cccccccccc}
\hline Variável & \multicolumn{4}{c}{ Alunas } & \multicolumn{4}{c}{ Alunos } \\
\hline & \multicolumn{2}{c}{ Mãe } & \multicolumn{3}{c}{ Pai } & \multicolumn{3}{c}{ Mãe } & \multicolumn{2}{c}{ Pai } \\
& N & $\%$ & N & $\%$ & N & $\%$ & N & $\%$ \\
\hline Média & 64 & 66,6 & 36 & 37,5 & 7 & 41,2 & 8 & 47 \\
$>2^{*}$ & 32 & 33,4 & 60 & 62,5 & 10 & 58,8 & 9 & 53 \\
$<2^{* *}$ & 96 & 100 & 96 & 100 & 17 & 100 & 17 & 100 \\
\hline Total & \multicolumn{4}{c}{ Legenda: *alta exigência; **baixa exigência } \\
\hline & Fonte: Narazaki HAM, et al., 2021.
\end{tabular}

No que tange ao sexo masculino, observou-se que $41,2 \%$ dos meninos consideraram alta responsividade materna, enquanto $58,8 \%$ consideram-na baixa. Já sobre os pais, $47 \%$ dos meninos consideram-na alta, enquanto $53 \%$ consideram-na baixa. Em linhas gerais, ambos - pai e mãe - com alta responsividade são minoria.

Após a análise dos sintomas e períodos do estresse juntamente com a relação parental para com os filhos, foi possível estabelecer uma relação entre ambos, utilizando a correlação de Spearman (Tabela 5).

Tabela 5 - Correlação entre escores de exigência/responsividade e estresse.

\begin{tabular}{ccc}
\hline & Desvio Padrão & Mediana \\
\hline Exigência & $r=0,064$ & $r=-0,098$ \\
& $p=0,502$ & $p=0,361$ \\
\hline Responsividade & $r=0,035$ & $r=-0,011$ \\
& $p=0,715$ & $p=0,917$
\end{tabular}

Legenda: ${ }^{*} r=$ coeficiente de correlação de Spearman; $p=p$ - valor; $n=113$ indivíduos.

Fonte: Narazaki HAM, et al., 2021.

Dessa forma, obteve-se uma correlação negativa entre responsividade $(r=-0,098)$ e a frequência dos sintomas estressores $(p=0,361)$, indicando que quanto menor o escore da responsividade maior é a frequência de sintomas de estresse.

Mediante a análise do período dos sintomas, também se obteve correlação negativa com a variável responsiva, na qual $(r=-0,011, p=0,917)$, ou seja, quanto menor o escore dessa variável maior a duração dos sintomas de estresse.

\section{DISCUSSÃO}

Baseado nos dados, observa-se a importante prevalência de estresse no sexo feminino, a qual é exclusiva se comparado com o grupo masculino, o que corrobora a hipótese, que está contida na literatura, de que a variável gênero influencia os níveis de estresse (MARQUES CP, et al., 2015; CALAIS SL, et al., 2003).

O grupo feminino, entre os 4 domínios do estresse, apresentou sintomatologia cognitiva e fisiológica. No caso da sintomatologia relativa à cognição, podem ser destacados, entre tais sintomas, perda de memória, prejuízos na fala e no pensamento. A incidência de tais problemas é extremamente limitante, pois prejudica o convívio do adolescente nos meios em que vive, além de prejudicar o estudo preparatório para o vestibular, visto que em ambas as situações a comunicação é extremamente importante, além de a memória ser fundamental para o aprendizado.

Mediante a sintomatologia fisiológica, é importante lembrar que, além de presente no grupo estudado, tal quadro de sintomas pode atrapalhar o cotidiano da adolescente, visto que os sintomas fisiológicos, como taquicardia, irritação gástrica, entre outros, podem afetar atividades realizadas pela adolescente, sendo ela 
acadêmica ou não. Caso seja acadêmica, pode prejudicar diretamente o estudo e rendimento, e caso seja sua forma de lazer, pode prejudicar de forma indireta o estudo, pois sem as formas de diversão e lazer, não há como descansar e se recuperar, atrapalhando, assim, o rendimento acadêmico. Ademais, a sintomatologia pode causar insegurança no adolescente, pois, no decorrer de suas atividades, tais sintomas a atrapalharão na execução e no bom resultado de determinada tarefa, prejudicando não só os estudos e o lazer, mas também a relação com a família (LIMA MMM, et al., 2021).

No que tange ao ambiente familiar, os dados coletados e interpretados apresentam que a relação entre genitores e filhos possui caráter altamente responsivo, ou seja, os pais apresentam ações de caráter acolhedor, dando suporte ao filho, com objetivo de favorecer o desenvolvimento e a autoafirmação do jovem. Vale salientar que a responsividade parental, em geral, é considerada alta, entretanto, somente no panorama geral do grupo, visto que, caso haja aprofundamento da amostra de estudantes, pode-se notar a presença de vestibulandos que consideram pais com nível baixo de auxílio, ou seja, deflagram relativa falta de comportamentos parentais que tentam de alguma forma apoiar e tranquilizar os filhos.

Nota-se, por exemplo, que mais da metade das meninas e dos meninos julgam seus respectivos pais como pouco responsivos, mostrando que, mesmo com tais familiares do grupo sendo majoritariamente classificados com nível alto de suporte, há grande parcela de alunos que consideram as relações de baixo teor responsivo, especialmente paterna.

Ainda relacionado ao ambiente familiar, infere-se que somente a presença de níveis altos de responsividade no panorama geral não gera garantia de ausência de estresse. Exemplificando, foram observados que mesmo com esse nível de alto apoio por parte dos progenitores, não necessariamente o nível de estresse será baixo, visto que o grupo foi classificado como alto apoio e mesmo assim houve prevalência de estresse no sexo feminino.

Ademais, no grupo feminino, o qual foi rotulado como estressado, pode-se observar a maior presença da mãe no âmbito familiar, visto que a maioria delas são altamente participativas e exigentes, enquanto os pais não são tão presentes nesses aspectos, pois são classificados pela maioria das meninas com baixos níveis de tais características. Como a responsividade demonstrou correlação negativa com sintomas e frequência de estresse, seria de suma importância que a maior parcela paterna fosse altamente resoluta, com finalidade de reduzir os fenômenos estressores.

Pode-se ainda observar que a ausência do caráter responsivo por parte dos progenitores pode ser responsável por um aumento da frequência e duração dos sintomas de estresse, portanto, é de suma importância haver no âmbito familiar alta responsividade, ou seja, ações que fomentem o apoio e a compreensão do adolescente, como apoiar e ouvir pensamentos dos filhos. Incentivar e raciocinar em conjunto são essenciais para determinação de uma menor prevalência de sintomas de estresse, visto que esses fatores de apoio auxiliam na forma com que o indivíduo avalia e responde ao fenômeno estresse, e, com base nessa avaliação, o jovem adulto pode lidar com adversidades que podem surgir no percurso da vida acadêmica de forma eficiente e adequada (SELYE H, 1965; STRAATMANN G, 2010).

Dessa forma, a correlação entre o ambiente familiar e os níveis de estresse corrobora a hipótese contida na literatura, na qual é afirmada a importância da relação entre os sintomas de estresse e a avaliação do adolescente a respeito do âmbito familiar no qual está inserido (JUSTO AP, 2005).

Em suma, a interação parental é significativamente importante para a modulação dos sintomas do estresse, no entanto, somente a responsividade parental não garante que não haja estresse, pois, como visto nos dados que correlacionaram o estresse com a interação de ambos os progenitores, somente $o$ alto suporte não afasta a possibilidade de haver estresse por completo, visto que as meninas consideraram seus progenitores majoritariamente altamente responsivos e mesmo assim seu escore de estresse ficou extremamente próximo ao considerado como grupo estressado; sendo, por esse motivo, juntamente com a presença de manifestações relacionadas com o estresse, classificado como tal. Portanto, urge a necessidade de pesquisas que busquem outros fatores que influenciem no estresse que não a responsividade (SALTON J, 1995; RÉ AHN, et al., 2004; LIMA MMM, et al., 2017). 


\section{CONCLUSÃO}

O estresse é muito prevalente em adolescentes, tornando-o um problema a ser monitorado, especialmente em mulheres, visto que houve prevalência no sexo feminino, que representou a maior parcela dessa amostra. O presente trabalho mostrou ainda que a influência dos pais pode trazer benefícios ao grau de saúde do jovem em relação à resposta ao estresse, pois, mediante o âmbito familiar, pode haver melhora nos sintomas de estresse caso haja uma relação de caráter mais responsivo, dessa forma, especialmente os pais das meninas necessitam de um maior nível de responsividade, visto que deflagrou-se um menor interesse nas atividades das filhas na maior parcela paterna, diferentemente das mães que em sua maioria demonstraramse altamente responsivas.

\section{REFERÊNCIAS}

1. AMARAL SCO, SANT'ANNA CC. Estresse em mães de crianças com asma: relato de pesquisa de um programa de redução do estresse. Revista Eletrônica Acervo Saúde, 2018; 11(1): 2346-2353.

2. ARAGÃO RC, et al. Cursinho Popular Emancipa: movimento de educação popular. Rev. Ed. Popular, 2015; 14(2): 8392.

3. MINISTÉRIO DA SAÚDE. Proteger e cuidar da saúde de adolescentes na atenção básica. Brasil, 2017. Disponível em: https://bvsms.saude.gov.br/bvs/publicacoes/proteger_cuidar_adolescentes_atencao_basica_2ed.pdf. Acessado em: 20 de junho de 2021.

4. CALAIS SL, et al. Diferenças de Sexo e Escolaridade na Manifestação de Stress em Adultos Jovens. Psicologia: Reflexão Crítica 2003; 16(2): 257-263.

5. DARLING N, STEINBERG L. Parenting style as context: an integrative model. Psychological Bulletin, $1993 ; 113$ : 487493.

6. GROLLI V, et al. Sintomas Depressivos e de Ansiedade em Adolescentes do Ensino Médio. Rev. Psicol. IMED, 2017; 9(1): 87-103.

7. JUSTO AP. A influência do estilo parental no stress do adolescente. Dissertação (Mestrado em Psicologia) - Pontifícia Universidade Católica de Campinas, Campinas, 2005; 116 p.

8. LANDSTEDT E, GADIN KG. Seventeen and stressed - Do gendeand class matter? Health Sociology Review, 2012; 21(1): 82-89.

9. LIMA MMM, et al. Perfil de manifestação de estresse em universitários: um estudo transversal. Revista Enfermagem Atual In Derme, 2021; 95 (33): e-021001.

10. LIPP MEN. Mecanismos Neuropsicofisiológicos do Stress: teoria e aplicações clínicas. 3 ed. São Paulo: Casa do Psicólogo, 2003; p. 17-18.

11. MACHADO SF, et al. Níveis de estresse em alunos de $3^{a}$ série do ensino médio. Universitas: Ciências da Saúde, $2011 ; 9(2): 35-52$.

12. MARQUES BAA. Impacto da divulgação das notas no Enem na concorrência entre escolas. Dissertação (Mestrado Profissional em Finanças e Economia) - Fundação Getúlio Vargas (FGV), São Paulo, 2013; 16 p.

13. MARQUES CP, et al. Fatores relacionados ao nível de estresse em adolescentes estudantes: uma revisão sistemática. Revista Salusvita, 2015; 34(1): 99-108.

14. PAGGIARO P, et al. Estresse e escolha profissional: um difícil problema para alunos de curso pré-vestibular. Contextos Clínicos, 2009; 2(2): 97-105.

15. PEREIRA TTSO, et al. O cuidado em saúde: o paradigma biopsicossocial e a subjetividade em foco. Mental, 2011; 9(17): 523-536.

16. RÉ AHN, et al. Stress e nível competitivo: considerações sobre jovens praticantes de futsal. Rev Bras Ci E Mov, 2004; 12(4): 83-87.

17. RIMOLI J, et al. Cursinhos comunitários e o direito à educação. Revista em Extensão, 2020; 18(2): 56-75.

18. SALTON J. Considerações a respeito do stress nas competições esportivas na infância e adolescência. Revista de Psiquiatria do Rio Grande do Sul, 1995; 17 (2): 160-163.

19. SANTOS FS, et al. Estresse em Estudantes de Cursos Preparatórios e de Graduação em Medicina. Revista Brasileira de Educação Médica, 2017; 41(2): 194-200.

20. SCHERMANN LB, et al. Estresse em adolescentes: estudo com escolares de uma cidade do sul do Brasil. Aletheia, 2014; 43-44: 160-173.

21. SCHÖNHOFEN FT, et al. Transtorno de ansiedade generalizada entre estudantes de cursos de pré-vestibular. Jornal Brasileiro de Psiquiatria, 2020; 69(3): sp.

22. SELYE H. Stress: a tensão da vida. 2. ed. São Paulo: Ibrasa, 1965.

23. SILVA LLT, et al. Profissionais de enfermagem de um serviço de urgência e emergência frente ao suicídio na adolescência. Revista Eletrônica Acervo Saúde, 2020; 12(10): e4042.

24. STRAATMANN G. Estresse, estratégias de enfrentamento e a percepção da imagem corporal em adolescentes: relações com o estado nutricional. Tese de Doutorado. Faculdade de Filosofia, Ciências e Letras de Ribeirão Preto Universidade de São Paulo, Ribeirão Preto, 2010. 26p.

25. TEIXEIRA MAP, et al. Refinamento de um instrumento para avaliar responsividade e exigência parental percebidas na adolescência. Avaliação Psicológica, 2004; 3: 1-12.

26. TRICOLI VAC. Escala de Stress para Adolescentes: criação e validação. Tese de Doutorado. Puc-Campinas, São Paulo, 2006.

27. VARGAS P, et al. Autonomia, Responsividade/Exigência e Legitimidade da Autoridade Parental: Perspectiva de Pais e Adolescentes. Psico-USF, 2017; 22(1): 23-34. 